\title{
Contribution à l'histoire de la radioprotection en France : l'environnement et la radioécologie de 1955 à 1965
}

\author{
R. COULON ${ }^{1}$
}

(Manuscrit reçu le 7 avril 2003, accepté le 23 juin 2003)

RÉSUMÉ Les études et recherches relatives à l'environnement, c'est-à-dire la radioécologie, ont débuté en France au milieu des années 1950, essentiellement au Commissariat à l'énergie atomique. La mise en ouvre de programmes nucléaires militaires, d'abord à l'étranger puis en France, a conduit à développer ces études, en raison des retombées dues aux essais atmosphériques américains et russes mais aussi des rejets des installations produisant les matières fissiles nécessaires (en France, le centre de Marcoule). Par la suite, le développement des utilisations civiles de l'énergie nucléaire a conduit à en faire une branche importante de la radioprotection. L'émergence de ces études, l'action des premiers acteurs, l'adaptation des structures, sont les éléments de l'histoire de cette discipline ; elles l'ont amenée, au milieu des années 1960, à son rythme de croisière. On présente l'histoire de la radioécologie durant cette période (1955-65), illustrée par les premières grandes études réalisées alors.

ABSTRACT Contribution to the history of radiation protection in France: environment and radioecology from 1955 to 1965.

Studies and researches concerning the environment, i.e., radioecology, started in France in the middle of the 50's, mainly at the French Atomic Energy Commission (CEA). The implementation of nuclear military programmes, first in foreign countries and then in France, led to the development of such studies, due not only to the fallouts of American and Russian atmospheric nuclear tests, but also to the releases from installations producing fissile materials (like in France, the center of Marcoule). Later, the development of civil uses of nuclear energy contributed to make radioecology an important branch of radiation protection. The elements of the history of this discipline are the emergence of radioecology studies, the contribution of the first involved researchers and the adaptation of structures that got their cruise speed in the middle of the 60's. In this paper, the history of radioecology during the period 1955-65 is presented and illustrated by the first large studies performed at that time.

\section{Préambule}

Les recherches sur la radiocontamination de l'environnement, rassemblées maintenant sous le nom de « radioécologie », sont nées en France avec les années

\footnotetext{
1 SFRP, B.P. 72, 92263 Fontenay-aux-Roses Cedex. France.
} 
1950. Entre les premiers travaux, fragmentaires et réalisés dans des structures plus ou moins bien adaptées, et les programmes actuels, l'évolution est considérable.

Pour aborder plus aisément cette évolution, il a paru utile de distinguer trois périodes dans la cinquantaine d'années considérée : la période 1955-65 qui a vu la naissance et les premiers développements des travaux, puis leur mise en place dans des structures adaptées; la période 1966-85 durant laquelle la radioécologie a atteint la maturité et a apporté les connaissances indispensables pour assurer la protection des populations vis-à-vis des risques potentiels associés au développement des programmes nucléaires; la période après 1986, année de l'accident de Tchernobyl, car les leçons tirées en matière de radioprotection de l'homme et de l'environnement ont beaucoup influencé les orientations des programmes de radioécologie. Ce travail concerne exclusivement la première de ces périodes, soit 1955-65.

\section{Introduction}

La radioprotection est née vers 1920. Au tout début, il s'agissait d'assurer la protection des personnes exposées en raison de leur activité professionnelle, notamment les médecins radiologues. Plus tardivement, est apparue la nécessité de se préoccuper aussi de la protection du public, en raison du développement des programmes nucléaires.

Le problème de la protection des personnes du public ne se pose pas dans les mêmes termes que celle des personnes professionnellement exposées. Pour ces dernières, l'exposition est en général liée à la source et/ou à la contamination du milieu fermé où elles exercent leur activité. Pour les personnes du public, c'est l'environnement, avec toute sa diversité et sa complexité, qui joue le rôle de vecteur et d'intermédiaire : entre l'émission dans l'environnement et l'atteinte du public se produisent des phénomènes d'accumulation, d'élimination, de transfert, de bio-concentration, qu'il s'agisse des sols, des eaux, des productions végétales et animales ou du milieu naturel. Pour limiter à des niveaux suffisamment faibles les conséquences d'une émission radioactive concertée dans l'environnement, unique ou répétée, ou pour prendre les mesures correctives nécessaires, il faut avoir une connaissance aussi complète et précise que possible du comportement à court, moyen et long terme, des radionucléides rejetés et des très nombreux paramètres qui les conditionnent.

Cette connaissance peut s'acquérir par deux voies distinctes et complémentaires : l'expérimentation, sous sa forme la plus simple en laboratoire, ou plus élaborée, dans des installations où puissent être simulées les conditions naturelles, ou encore réalisée in situ; l'observation in situ, après des contaminations réelles, accidentelles ou contrôlées. 
L'exposition subie par les personnes professionnellement exposées peut être mesurée directement, notamment par la dosimétrie personnelle ou d'ambiance, l'anthropogammamétrie, ou les mesures sur les excrétas ; par contre, celle qui est subie par les personnes du public ne peut, sauf cas très particuliers, qu'être calculée à partir des mesures faites dans l'environnement (air, eau, denrées alimentaires, etc.) : les programmes de surveillance du milieu ont une grande importance.

Tel était le difficile challenge qui s'offrait à ceux qui n'étaient pas encore des «radioécologistes» ni même des «écologistes", mais qui ont fait de la radioécologie... sans le savoir. Le terme «écologie » est employé, sans doute, depuis le milieu des années 1950 , celui de « radioécologie » depuis la fin de cette période (vers 1960). A. Grauby, l'un des pionniers, rappelle que cette terminologie a été importée des États-Unis, plus précisément du centre d'Oak Ridge où une unité de radioécologie avait été créée au sein de la Health Physics Division (Grauby, 2002).

\section{Les événements qui ont motivé les études sur l'environnement}

Lorsqu'est créé en 1951, par le Commissariat à l'énergie atomique, le Service de protection contre les rayonnements (SPR), rien dans ses attributions ne porte de façon explicite sur les aspects environnementaux $[a]^{*}$. Pourtant, ces problèmes se sont déjà posés, du fait de la réalisation des programmes nucléaires militaires aux États-Unis et en URSS dès les années 1940 (Foulquier, 2000). Il est vrai que le secret lié au caractère militaire de ces opérations, l'extrême discrétion des soviétiques et le fait que l'embargo pratiqué vis-à-vis de la France par les ÉtatsUnis et la Grande Bretagne n'est pas encore totalement levé, sont autant d'éléments qui sont à l'origine de la rareté de l'information en la matière.

Lorsque le Dr H. Jammet, à qui la direction du SPR avait été confiée, est dépêché auprès du Comité scientifique des Nations-Unies pour l'étude des effets des rayonnements ionisants (UNSCEAR) en 1956, pour participer à l'élaboration de son premier rapport, il prend conscience de l'importance des problèmes posés par la contamination radioactive de l'environnement et du retard de la France dans ce domaine. Dans le premier rapport UNSCEAR publié en 1958 (UNSCEAR, 1958), on trouve en effet des données sur la contamination de nombreux produits alimentaires par ${ }^{90} \mathrm{Sr}$, ${ }^{137} \mathrm{Cs}$ et ${ }^{131}$ I fournies par divers pays (États-Unis, Grande Bretagne, Japon, URSS, etc.), mais aucune donnée pour la France. Il faut attendre les rapports de 1962 et 1964 pour trouver des données françaises. Cette situation

\footnotetext{
* $C f$. Notes en fin d'article.
} 
lui permet de convaincre les autorités de lui donner les moyens nécessaires pour combler le retard en développant les contrôles et les études appropriés.

Cette nécessité est d'autant plus évidente que les longues séries d'essais nucléaires atmosphériques réalisées dès 1945 par les États-Unis et dès 1949 par 1'URSS ont entraîné, dans la seconde moitié des années 1950, une contamination radioactive de l'environnement très significative, y compris en France, avec un premier pic atteint en 1959 et un second, plus important, en 1963 (Coulon, 1992). L'inquiétude suscitée était d'autant plus légitime que les conséquences à moyen et long terme de cette contamination étaient difficiles à apprécier en raison du manque de connaissances.

À cette époque, la France décide de se doter aussi de l'arme atomique et de développer un programme nucléaire militaire, qui va entraîner la création du centre de Marcoule avec l'implantation de trois réacteurs (1956 à 1959) et d'une usine de retraitement du combustible (1958) destinée à produire le plutonium nécessaire à la fabrication des armes. Cet établissement va rejeter des effluents radioactifs dans le Rhône et l'on sait combien est importante l'utilisation agricole des eaux de ce fleuve, prélevées en aval de Marcoule (Fourques), que ce soit pour les rizières de Camargue ou pour l'irrigation d'une vaste zone de production légumière du BasRhône-Languedoc.

Il faut ajouter que le développement industriel, qui a fortement marqué la première moitié du $20^{\mathrm{e}}$ siècle, avait déjà eu pour conséquence le rejet de polluants chimiques dans l'environnement, notamment les eaux de surface, donc une certaine contamination des terres cultivées. Il en est résulté une levée de boucliers dans le monde agricole et une vive tension entre ce milieu et le monde industriel. Ce problème a fait l'objet d'une séance de l'Académie d'agriculture, le 25 juin 1958 (Académie d'agriculture, 1958) ; le compte rendu fait état de la « grande émotion du monde rural » et de «l'hostilité manifestée vis-à-vis de certains groupements industriels », se traduisant par des affiches et des tracts demandant une «action directe » contre les auteurs des pollutions. Le débat porte sur des sujets encore très actuels, comme l'acceptabilité du risque, la nécessité de ne pas risquer d'entraver un développement utile pour la société, ou encore la difficulté à légiférer.

Cette inquiétude gagne le monde de la pêche en raison de la mise en service, en 1956, de l'usine de retraitement des combustibles de Windscale (Angleterre), qui rejette ses effluents radioactifs en mer d'Irlande, zone régulièrement fréquentée par des pêcheurs français. Il ne faut pas oublier l'accident qui, en 1957, s'est produit dans cet établissement et dont les conséquences, bien que limitées, au niveau des productions agricoles (essentiellement le lait) ont mis en évidence le fait que ce type d'événement pouvait bel et bien arriver. 
Il ne fait aucun doute que ce sont les aspects militaires de l'utilisation de l'énergie atomique qui ont été le moteur principal de la naissance et des premiers développements des études radioécologiques, avec une sensibilisation particulière du milieu agricole. Mais il est tout aussi évident que c'est l'intensification des utilisations de l'énergie nucléaire dans de nombreux domaines, et tout particulièrement celui de la production d'énergie à des fins civiles, qui a soutenu et amplifié ce développement. La création de nombreux sites du CEA et de l'EDF a nécessité la mise en place d'une méthodologie rationnelle et écologique des études d'impact. La création des centres de production EDF, avec leurs rejets dans les grands fleuves, est pour beaucoup dans le développement de la radioécologie des eaux continentales, et celle de l'usine de retraitement des combustibles de La Hague dans le développement de la radioécologie marine. Enfin, la perspective de la réalisation d'essais nucléaires atmosphériques en Polynésie a nécessité de se préoccuper des problèmes d'environnement qui allaient inéluctablement se poser.

\section{Les structures et les hommes}

La radioécologie, et d'une façon générale les études et recherches relatives à la radiocontamination de l'environnement, sont nées et se sont développées presque exclusivement au Commissariat à l'énergie atomique. D'autres acteurs (EDF, universités, etc.) sont intervenus, mais de façon relativement peu importante, au moins au cours de la première période traitée ici.

En 1956, l'éclatement du SPR, (voir plus haut ses attributions), marque le début de la recherche de structures mieux adaptées au développement de la radioprotection, et notamment de la radioécologie. Le SPR se scinde en deux unités $[b]$, le service d'hygiène atomique et de radiopathologie (SHARP), confié à H. Jammet, et le service de contrôle des radiations et de génie radioactif (SCRGR), confié à $\mathrm{F}$. Duhamel. La note $[b]$ définit la mission de ces deux services.

Le SHARP est chargé de tout ce qui concerne le milieu vivant en matière d'hygiène radiologique, notamment vis-à-vis des travailleurs exposés aux rayonnements. En matière d'environnement, il est chargé d'effectuer des recherches sur les fixations biologiques des radioéléments pouvant contaminer les effluents rejetés par les installations nucléaires, et de surveiller la contamination radioactive des plantes, des algues, des poissons... exposés à ces effluents. En ce domaine, il s'adresse au SCRGR pour susciter la création d'appareils de mesure, ou leur perfectionnement.

Outre la tâche qui vient d'être évoquée, le SCRGR est chargé des contrôles aux alentours des établissements du CEA, selon les prescriptions générales ou les 
demandes particulières formulées par le SHARP, et de transmettre au chef du SHARP les résultats de ces mesures.

Déjà peu claire sur le papier, cette distinction l'est encore moins dans les faits, et les débordements sont assez nombreux : il n'est pas évident de traiter des problèmes concernant le sol en faisant abstraction de la couverture végétale ou concernant l'eau sans considérer la flore et la faune aquatiques. L'émulation fut, sans doute, utile pour le développement des connaissances.

Le SHARP s'empare immédiatement de la mission de contrôle de la contamination biologique due aux rejets d'effluents, et crée un "groupe de surveillance » destiné à assurer les trois opérations nécessaires : prélèvements, mesures, interprétation des résultats. Très vite, le chef du SHARP adresse au haut commissaire une note, $[c]$, dans laquelle il propose l'organisation de ce groupe, et détaille les moyens nécessaires en personnel, locaux, matériels et crédits. On notera que, parmi les personnes demandées, figure un écologiste. La note se réfère aussi aux éventuels rejets d'effluents radioactifs dans la Seine et dans le Rhône, qui ne seraient autorisés qu'à la condition expresse que la contamination susceptible d'en résulter soit contrôlée, et que la radioactivité naturelle soit préalablement mesurée, afin de servir d'élément de référence.

En 1957, sont créés au sein du SHARP $[d]$ un groupe "contamination alimentaire » sous la responsabilité du Dr G. Michon, vétérinaire, et un groupe «radiotoxicologie», sous la responsabilité de L. Jeanmaire, pharmacien. G. Michon est en charge de toutes les études liées à l'environnement. Il développe une collaboration avec l'Institut national de la recherche agronomique (INRA) et avec le Centre national de recherches zootechniques (CNRZ), ainsi qu'avec l'Institut scientifique et technique des pêches maritimes, cette dernière collaboration ayant servi d'amorce aux études de radioécologie marine. L. Jeanmaire met sur pied un laboratoire de mesure de faibles radioactivités dans les échantillons biologiques. Avec lui, R. Coulon, agronome, et C. Madelmont, vétérinaire, recrutés pour développer le volet «contrôle» en liaison avec le Ministère de l'agriculture, mettent au point une méthodologie de type séquentiel, nécessaire pour mesurer avec des moyens encore limités la contamination d'échantillons très nombreux et variés de la chaîne alimentaire.

Au SCRGR, F. Duhamel charge, à l'automne 1956, A. Grauby, universitaire, et C. Candillon, agronome, de lancer, au sein d'un groupe "écologie», un programme d'étude de la dispersion des radionucléides dans l'environnement. P. Bovard, également agronome, rejoint le groupe en 1958, alors que C. Candillon le quitte en 1959. Les travaux portent sur l'impact des premières installations nucléaires sur l'environnement, et les thèmes de recherche sur la dispersion des 
radionucléides en milieu aquatique, et leur diffusion dans les sols. Le SCRGR s'associe également à l'INRA, via le Centre d'étude du machinisme agricole, du génie rural et des eaux et forêts (CEMAGREF).

En 1961, le SHARP est dissout. Il est remplacé par le Département de protection sanitaire (DPS), [e], toujours dirigé par H. Jammet [f]. Le DPS est structuré en trois entités $[g]$ : un service d'hygiène atomique, un service de radiopathologie, une section de contrôle sanitaire. Celle-ci, dirigée par G. Michon [h], est chargée, entre autres, «d'effectuer ou faire effectuer toutes études et recherches permettant d'évaluer les retombées radioactives et la contamination radioactive du milieu biologique, de la chaîne alimentaire et des personnes, et de toutes études et recherches relatives à cette contamination ». La section de contrôle sanitaire est structurée en trois groupes [i]. Un groupe «analyses et mesures », placé sous la responsabilité de L. Jeanmaire, est chargé de l'exécution des mesures radiochimiques et physiques sur échantillons biologiques, ainsi que des recherches associées. Un groupe "organisation et interprétation », confié à R. Coulon, a pour tâche la mise en cuvre d'une surveillance nationale de la contamination des produits alimentaires, avec le Ministère de l'agriculture et, ultérieurement, de la centralisation et de l'interprétation des résultats. Enfin, un groupe "radioécologie », confié au Dr J. Ancellin, vétérinaire, débute les études en milieu marin.

C'est là que la radioécologie commence à se construire. En milieu marin, les premiers travaux sont menés avec le Pr Y. Fontaine, du Museum d'histoire naturelle, ainsi qu'avec le groupe d'études atomiques de la marine nationale. Ils sont liés aux études qui précèdent l'implantation de la future usine de retraitement des combustibles sur le site de La Hague. Le Pr M. Avarguès, adjoint de H. Jammet, suit et appuie très fortement le développement de ces travaux. Dans le même temps, G. Michon prépare la création de la future radioécologie continentale: en 1963, i1 recrute L. Foulquier, l'un des premiers diplômés d'écologie, et J. Delmas, agronome. Ils sont dépêchés sur le site de Cadarache avec pour missions respectives de créer un laboratoire d'hydrologie des eaux douces et un laboratoire de radioécologie terrestre.

Il faut mentionner l'existence et l'intégration d'un laboratoire de métrologie issu du LDG (Laboratoire de détection et de géophysique du Pr Y. Rocard), implanté sur le site de la faculté des sciences d'Orsay, et dirigé par J.C. Philippot. Spécialisé dans le développement de mesures ultra-fines de radioactivité, ce laboratoire va aussi mettre en place un petit réseau de prélèvements atmosphériques (air et eau).

La dualité entre SHARP et SCRGR se poursuit entre DPS et SCRGR, avec une nette pré-éminence du rôle du DPS dans le domaine de la contamination du milieu. 
Le SCRGR reste cependant responsable en matière de sites ; il est restructuré en $1962[j]$ selon quatre sections, dont une section «étude et contrôle des sites », dirigée par A. Menoux.

En 1964, le SCRGR est dissout [ $k$ ]. Ses missions, ses moyens, passent dans d'autres unités, le DPS reprenant tous les aspects relatifs à l'environnement ainsi que la centralisation, l'interprétation et la diffusion des résultats des mesures de surveillance effectuées dans les centres du CEA. P. Bovard et A. Grauby rejoignent le DPS. Tous les éléments de base de la radioécologie se trouvent enfin rassemblés au sein d'un même département. Ils sont répartis en trois groupes : un groupe de radioécologie marine (J. Ancellin), implanté sur le site de La Hague, et déjà présent dans la section de contrôle sanitaire ; un groupe de radioécologie continentale (A. Grauby, J. Delmas, adjoint [l]), sur le site de Cadarache ; un groupe d'études atmosphériques (A. Doury), implanté à Guyancourt.

Les missions du nouveau groupe de radioécologie continentale sont précisées par le chef du DPS $[\mathrm{m}]$, à savoir l'étude des processus de transfert et de concentration des radionucléides en milieu continental, les études écologiques relatives aux nouveaux sites et aux sites existants, les études des effets de l'irradiation et de la contamination sur la biosphère continentale.

En 1965, les trois groupes sont réunis en un «ensemble de radioécologie » $[o]$, dont P. Bovard assure la coordination $[p]$. La même année, cet ensemble est transformé en section de radioécologie $[q]$; sa mission est d'effectuer ou de faire effectuer toutes études et recherches sur les transferts dans le milieu ambiant et la chaîne alimentaire des radionucléides et de toutes substances susceptibles d'intéresser le CEA ; l'action des produits désignés ci-dessus dans le milieu ambiant, flore et faune ; la fixation des niveaux des paramètres intervenant dans l'estimation de leur dissémination dans l'environnement. La section de radioécologie est confiée à P. Bovard $[r]$; elle s'étoffe par le recrutement de nombreux spécialistes de l'environnement (agronomes, vétérinaires, universitaires). En 1977, la section deviendra le service d'études et de recherches sur l'environnement, que dirigera A. Grauby.

Toujours au CEA, la mise en œuvre du programme d'expérimentation d'engins nucléaires en Polynésie entraîne la création de structures adaptées aux importantes nécessités de la surveillance, ce, bien entendu, en étroite liaison avec le Ministère des armées. Comme ces structures joueront à plein temps leur rôle à partir de 1966, début des essais atmosphériques, elles seront traitées avec la seconde période (1966-85) de l'histoire de la radioécologie.

En dehors de ces structures, d'autres unités du CEA ont des activités liées à l'environnement, mais dans des domaines différents, soit parce qu'il s'agit de recherches plus fondamentales, par exemple des études de biologie végétale 
utilisant des indicateurs radioactifs pour l'étude de certaines fonctions (ex. : la photosynthèse), soit au contraire, d'études très appliquées, utiles en agronomie (ex. : l'utilisation des engrais); c'est le cas du Département de biologie à Saclay, et celui du Service de radioagronomie, à Cadarache.

En 1959 est créé le laboratoire de biologie végétale, sur le centre CEA de Grenoble. Il est dirigé par P. Ozenda, professeur à la faculté des sciences de Grenoble ; le personnel est mixte, CEA et CNRS. L'un des groupes du laboratoire, "physiologie végétale et radioécologie» (Mlle S. Gagnaire) s'intéresse à l'utilisation des végétaux comme indicateurs de contamination radioactive; il étudie la contamination radioactive d'une biocénose dans un écosystème reconstitué.

Enfin, à l'extérieur du CEA, rappelons-le, des études expérimentales sont réalisées au museum d'histoire naturelle par le Pr Fontaine, sur certaines espèces de la faune aquatique.

Cet article est consacré à la situation en France. Il faut cependant signaler qu'à l'étranger, les études et recherches sur la contamination radioactive du milieu ont débuté plus tôt qu'en France. Mais la motivation de ces études a été la même, à savoir le développement de programmes nucléaires militaires à partir des années 1940, avec les sites bien connus de Hanford et d'Oak-Ridge pour les États-Unis, Tcheliabinsk pour l'Union soviétique. Des publications scientifiques sur la contamination radioactive des systèmes aquatiques et des sols sont apparues à partir de 1945.

\section{Les études principales dans leur chronologie}

Bien que les études sur la contamination radioactive de l'environnement aient débuté vers 1955, ou un peu avant, les publications scientifiques sont très rares avant 1960, mais leur nombre augmente rapidement, ensuite : l'importance de la confidentialité qui régnait au CEA, et, peut être, le moindre intérêt accordé par les chercheurs aux publications en sont sans doute la cause. Les rapports annuels du CEA ne comportent une rubrique «hygiène atomique » qu'après 1960 et une rubrique «contrôle sanitaire et radioécologie » qu'à partir de 1965. Les quelques études sommairement décrites ici n'ont pour objet que d'illustrer et concrétiser les premiers travaux réalisés en la matière.

\subsection{Le contrat CEA-INRA : première étude importante de radioécologie}

Survenant dans la situation évoquée plus haut, le problème de la pollution radioactive ne peut qu'être regardé d'un mauvais œil par le milieu agricole. 
C'est sans doute la raison pour laquelle il est l'objet de la première étude radioécologique entreprise en 1956 avec l'INRA dans le cadre d'un contrat passé avec le SHARP sous l'impulsion de J. Debiesse, directeur du centre d'études de Saclay, et géré par G. Michon pour le CEA et par G. Barbier pour l'INRA. G. Barbier, agronome, est directeur de recherche à la station centrale d'agronomie de Versailles ; il est l'auteur de nombreux travaux sur le comportement d'éléments minéraux dans les sols et leur transfert aux plantes. L'étude devait répondre à des questions essentielles: que se passe-t-il en cas d'apport unique ou répété d'éléments à vies longues $\left({ }^{90} \mathrm{Sr},{ }^{137} \mathrm{Cs}\right)$ sur un sol ? Quels seront les transferts aux cultures? Quelles seront les influences de la nature du sol, du mode d'apport, du climat, de la nature du végétal ?

Le programme débute en 1956. Pour des raisons évidentes, on commence par l'étude du transfert du ${ }^{137} \mathrm{Cs}$ au riz. Dès 1957 , des parcelles expérimentales sont contaminées selon différents modes d'apport, dans les stations d'agronomie de Versailles, Avignon, Toulouse, Bordeaux, Narbonne, et mises en culture avec des productions légumières diverses, une prairie permanente (Versailles) et une vigne (Narbonne). Ces travaux ont donné lieu à de nombreuses publications (Académie d'agriculture, 1963; Barbier et Michon, 1959; Barbier et Michon, 1960 ; INRA/SHARP, 1961 ; INRA/DPS, 1962).

La rigueur des mesures de protection n'avait que peu de rapport avec celle des mesures prises actuellement. Les parcelles sont clôturées et des panneaux indiquent le danger; de plus, les contaminations apportées restent limitées $\left(25 \mu \mathrm{Ci} / \mathrm{m}^{3}\right.$, soit $10^{6} \mathrm{~Bq} / \mathrm{m}^{3}$, environ). Cependant, ces précautions n'ont pas permis d'éviter certaines intrusions. Autre pratique surprenante, tout au moins pour la parcelle de Versailles, celle qui consistait à stocker les fioles contenant les reliquats des solutions radioactives diluées dans un puisard situé sur la parcelle elle-même, donc facilement accessibles par des étrangers.

\subsection{Les premières études d'impact et l'acquisition de connaissances « in situ »}

Entre 1955 et 1965, la radioécologie (sans cette appellation) débute dans le groupe «écologie » du SCRGR, avec un objectif très pratique: les études d'environnement qui doivent précéder la création des sites nucléaires. On avait parfaitement conscience de la nécessité d'avoir une bonne connaissance de l'environnement dans lequel des installations nucléaires allaient fonctionner en rejetant des effluents radioactifs. L'expérience aidant, sont jetées les bases d'une méthodologie générale applicable à toutes les études de sites, dont le nombre allait inévitablement se multiplier. Au fil du temps, cette méthodologie s'est affinée et enrichie. Elle permet d'explorer l'environnement dans toute son hétérogénéité, 
de mettre en évidence l'ensemble des voies de transfert, en particulier celles qui sont spécifiques du site considéré, de quantifier ces transferts, enfin d'établir un « point zéro » radiologique.

Les tout premiers travaux ont porté sur l'impact de l'usine d'extraction du plutonium du Fort de Châtillon (futur centre d'études de Fontenay-aux-Roses) sur son environnement, encore peu urbanisé. Ils se sont ensuite étendus au sud de l'îlede-France, autour des sites existants ou en voie d'implantation, comme Le Bouchet, Saclay, Bruyère-le-Châtel... Expérimenter et prélever dans l'usine de plutonium du Fort de Châtillon relevait d'un pari fou, mais passionnant, qui choquerait très certainement les « chastes oreilles " des radioprotectionnistes actuels. Malgré ce contexte très particulier, où les observations des profils de sol de la butte sur laquelle était bâtie l'usine se font en scaphandre, stylo dosimètre dans la poche, avec un marteau piqueur et une tarière, les grandes lignes du cycle du césium, du strontium et des actinides dans les sols sont identifiées : compte tenu du statut particulier de l'usine du plutonium, ces travaux ne purent être publiés, et ne furent l'objet que de notes internes.

Voici une anecdote : au sommet de la pyramide de l'écosystème qui proliférait dans l'usine, se trouvait un chat. Ce chat bloquait systématiquement les instruments de contrôle, ce qui ne l'empêchait pas de posséder une vitalité exceptionnelle. Sa capture, pour raison de sécurité, eut lieu au laboratoire de contrôle radiochimique. Il est regrettable que les moyens d'investigation de l'époque aient été limités, car ce chat était certainement un excellent échantillon pour l'observation du métabolisme des produits de fission et surtout des actinides. Au départ des équipes du Fort de Châtillon pour Marcoule, les études « écologiques » se sont portées sur la future centrale EDF de Chinon (1958) et sur le site de Cadarache (1959).

\subsection{Les premières études expérimentales sur la dispersion des radionucléides dans les sols}

Parallèlement au contrat SHARP/INRA, le SCRGR s'est associé au génie rural pour développer des expériences sur la migration des radionucléides dans les sols. Une équipe mixte est créée avec A. Grauby (SCRGR) et J. Bourrier (ingénieur en chef du génie rural). Le site expérimental est celui du génie rural et du machinisme d'Antony (région parisienne), associé à celui de Vergière (Bouches-du-Rhône). Après la mise au point, en 1958, du «cube Vergière ", un micromonolithe permettant d'étudier la migration des radionucléides dans les profils de sols, une expérimentation sur de petits lysimètres est mise en place à Antony. Elle porte notamment sur le couple ruthénium-rhodium, choisi en raison de sa présence majeure dans les effluents de Marcoule et de l'absence de connaissances sur son 
comportement dans l'environnement (Bovard et al., 1961). Ces travaux concluent à une extrême mobilité dans les profils de sol, aux faibles niveaux rencontrés dans les productions végétales (Bourrier et al., 1960a, 1960b ; Bovard et Grauby, 1960).

\subsection{Les premières études concernant le milieu aquatique}

À partir de 1959, à l'initiative de P. Bovard, des études concernant le milieu des eaux douces sont lancées sur trois thèmes : hydrogéologie sous la responsabilité de A. Barbreau, hydrologie sous la responsabilité de Mlle Cattan, hydrologie de surface sous la responsabilité de A. Grauby. Ces études se sont prolongées bien au-delà de 1960. C'est notamment le cas des étangs de Saclay, reconstitués en laboratoire sous forme de micro étangs pour y réaliser des études de dispersion, ainsi que de certains cours d'eau comme la Juine, qui traverse le site du Bouchet. Les sites de Saclay, de Cadarache, de Chooz ont fait l'objet d'études hydrogéologiques approfondies, avec l'aide du BRGM. Enfin, la mesure en continu des radionucléides dans les cours d'eau, les étangs ou encore les réseaux d'eaux usées, a bénéficié d'une attention particulière (Bovard et Grauby, 1962). En 1960, avec l'aide d'une jeune entreprise (SEIN, P. Rimbault), sont réalisés la station d'essai de Saclay, la salle souterraine de contrôle des égouts de Fontenay, les premiers réseaux de contrôle de sites du CEA et de sites miniers, avec l'équipe de J. Pradel.

\subsection{La première thèse de radioécologie}

Tandis qu'après 1960 se poursuivent les travaux du contrat CEA/INRA, le SHARP accueille une doctorante, Mme Ferron, qui prépare un doctorat de spécialité en physiologie végétale, sur le thème « absorption du césium par l'orge. Influence de sa rétention dans le sol. Action compétitive du potassium ». Son directeur de thèse est G. Barbier, et les travaux se font chez G. Michon, à Fontenay. Les cultures sont réalisées dans des bacs disposés dans le grenier de l'une des antiques constructions en bois du Fort. Ce ne sont pas des conditions de travail idéales, sauf pour les nombreux chats qui peuplent le centre et qui, un jour, ont trouvé-là une herbe tendre très à leur goût. Il a fallu, non sans mal, capturer les délinquants afin de s'assurer qu'ils n'étaient pas trop contaminés. Cette première thèse de radioécologie donne lieu à publication (Ferron, 1964).

\subsection{Des réseaux de surveillance de la radioactivité dans l'environnement}

À la fin des années 1950 , le Service central de protection contre les rayonnements ionisants (SCPRI), créé auprès de l'Institut national d'hygiène (arrêté du 13 novembre 1956), développe un réseau national de contrôle de la contamination radioactive de l'environnement. Compte tenu des moyens encore limités dont il 
dispose, ce réseau concerne essentiellement le milieu physique (poussières atmosphériques, précipitations). À partir de 1962, des prélèvements de lait sont effectués dans les divers départements, mais leur nombre - donc leur représentativité - est assez peu important.

Le SCPRI revendique cette mission de contrôle au titre de la santé publique ; cependant, le CEA, seul ou avec le concours du ministère de l'agriculture, décide de mettre en place une surveillance de la contamination radioactive des productions agricoles. L'objectif est de mieux apprécier les problèmes environnementaux créés par ses installations et par les retombées dues aux essais nucléaires atmosphériques ; l'objectif est aussi de développer des connaissances sur les modalités de la radiocontamination de ces productions. Pour faire face au très grand nombre de mesures à faire et à la diversité des échantillons prélevés, une méthodologie adaptée est mise au point (Coulon et al., 1963).

(a) Le premier réseau de contrôle mis en place couvre toute la zone du BasRhône-Languedoc, où les eaux du Rhône sont utilisées pour l'irrigation des cultures. En liaison avec la Compagnie nationale du Bas-Rhône-Languedoc, un certain nombre de producteurs concernés sont choisis afin que, parallèlement au contrôle de l'eau d'irrigation, soit effectué un contrôle mensuel d'échantillons de divers légumes. La prise de contact avec ces producteurs avait été considérée comme très délicate, en raison de l'attitude hostile du milieu agricole régional visà-vis de Marcoule. En réalité, toutes les personnes concernées ont accepté très facilement de participer, considérant que ce contrôle serait une bonne garantie de la qualité de leur production. Les résultats de ce contrôle ont été régulièrement publiés, mais n'ont jamais donné lieu à une exploitation scientifique rendue impossible par le fait que les très faibles contaminations mesurées étaient dues aux retombées, comme le montraient les échantillons témoins.

(b) Le second réseau de contrôle, beaucoup plus important, est à l'échelle nationale. Il vise précisément les retombées des essais nucléaires atmosphériques : les mesures faites ça et là montraient, effectivement, des contaminations par le mélange des produits de fission, notamment ${ }^{131} \mathrm{I},{ }^{90} \mathrm{Sr},{ }^{137} \mathrm{Cs}$; elles étaient loin d'être négligeables. Outre la nécessité d'avoir une vision complète et suivie de ce qui pouvait poser problème, il était tentant de chercher à utiliser les résultats du contrôle pour en tirer de précieux renseignements sur le comportement et le transfert des radionucléides, c'est-à-dire des connaissances radioécologiques. Pour cela, il fallait que les résultats des mesures soient superposables et représentatifs, ce qui nécessitait un échantillonnage au niveau de la production.

La mise en place est faite avec le ministère de l'agriculture, dans le cadre d'une convention avec la Direction de la production et des marchés, dont le directeur était M. Cointat, agronome qui fut plus tard ministre de l'agriculture. 
Cette direction rassemblait tous les services aptes à jouer un rôle : le service de la protection des végétaux, pour la collecte des échantillons de fruits et de légumes ; le service de la répression des fraudes pour la centralisation, la préparation des échantillons composites, leur préparation physique, les premières mesures d'activité globale, pour ces échantillons de fruits et de légumes; les services vétérinaires pour toutes les opérations précédentes au niveau du lait et parfois des viandes. En ce qui concerne les céréales, une organisation similaire fut mise en place avec l'Union nationale des coopératives agricoles de céréales (UNCAC).

Les échantillons sont prélevés à la production, avec une fréquence leur assurant une bonne représentativité dans le temps. Leur regroupement se fait sur une base mensuelle et sur une base régionale, la région étant définie par rapport à des critères d'homogénéité des conditions climatiques, pédologiques, agronomiques et zootechniques. On aboutit à un certain nombre d'échantillons composites mensuels régionaux pour chaque catégorie de produits, sachant qu'une fraction de chaque échantillon initial est conservée pour le cas où un résultat anormal obtenu sur un échantillon composite demanderait une recherche d'origine. Au total, près de 10000 échantillons initiaux sont prélevés annuellement (Coulon, 1966). Enfin, les laboratoires des services vétérinaires et du service de la répression des fraudes disposent d'un matériel permettant des mesures immédiates d'activité globale, fort utiles pour détecter rapidement une éventuelle situation anormale, et permettre une intervention plus rapide.

Ce système disparaît peu à peu vers la fin des années 1970. Le SCPRI dispose alors de moyens importants ; il a accru considérablement ses réseaux de contrôle, alors que le ministère de l'agriculture, peu argenté et confronté à de nombreux problèmes sanitaires, cède du terrain. Il faut dire que les niveaux de contamination sont très faibles, et que l'intérêt radioécologique est devenu moins important. Bien que certains laboratoires, notamment les laboratoires centraux du service de la répression des fraudes et des services vétérinaires, aient conservé quelques compétences dans les mesures de radioactivité, il ne reste pas grand chose de cette organisation quand survient l'accident de Tchernobyl en 1986 : il fallut remettre en place des moyens, pour permettre aux mêmes structures de jouer un rôle qui n'aurait jamais dû être abandonné.

(c) À cette surveillance des produits agricoles s'ajoutent d'autres types de prélèvements, en particulier des prélèvements mensuels de régime alimentaire complet, effectués dans un grand lycée parisien, et surtout des prélèvements d'os humains effectués, dans des conditions réglementaires douteuses, dans plusieurs hôpitaux parisiens et bordelais. Avec les nombreuses mesures faites sur des prélèvements atmosphériques, d'eau de pluie et de sols, on a pu ainsi disposer d'un ensemble de données qui, associées à d'autres considérations, par exemple des 
données météorologiques ou pédologiques, ont permis d'établir une base de connaissances extrêmement précieuse pour l'avenir (Coulon, 1972 ; Coulon et Madelmont, 1969).

D'autres études, sur des points précis, ont pu être menées in situ, en profitant du «marquage » par les retombées. Dans le domaine de la contamination des céréales, des observations sont effectuées sur les champs expérimentaux de 1'UNCAC en région parisienne. Elles consistent à couvrir certaines parcelles pendant les différents stades végétatifs, de la montaison à la récolte, afin de les soustraire aux retombées et à déterminer le degré de sensibilité de chaque stade vis-à-vis de la contamination à la récolte, ce qui peut être très important en cas d'accident nucléaire (Coulon, 1969).

(d) Dans le domaine de la contamination des produits animaux, des observations du même type sont également effectuées. En 1962, sous la direction de G. Michon, et en liaison avec la station de physiologie animale du Centre national de recherches zootechniques, la contamination d'un troupeau de bovins alimenté avec des fourrages verts naturellement exposés aux retombées, est étudiée et suivie; l'absorption, l'excrétion et le transfert de ${ }^{131} \mathrm{I}$ au lait et à la viande sont quantifiés (Michon et Jeanmaire, 1963). Cette expérience sera renouvelée en 1987, à la suite de l'accident de Tchernobyl, par le Dr F. Daburon, vétérinaire, dans une structure de recherche mixte CEA(DPS)/INRA(CNRZ) à Jouy-en-Josas. Des vaches laitières sont alimentées à partir de fourrages récoltés dans la Drôme et naturellement contaminés par les retombées dues à l'accident. On suit le transfert des césium-134 et 137 dans le lait et la viande, et, pour certaines d'entre elles, dans leur descendance (Daburon et al., 1987).

La structure mixte mentionnée ci-dessus est le laboratoire-étable de radiobiologie appliquée (LERA) dont la création sur le site de Jouy-en-Josas fut décidée en 1960 dans le cadre d'un contrat CEA(DPS)/INRA(CNRZ). La mise en place de ce laboratoire prit un certain temps puisqu'il n'est véritablement entré en fonctionnement qu'en 1966, sous la responsabilité du Dr P. Nizza, vétérinaire. Cela est dû au fait qu'il s'agissait d'une structure assez complexe ; fait curieux, il y eut aussi le retard apporté par la présence d'un escarpement rocheux à l'endroit prévu pour la construction, et à la difficulté de se procurer les explosifs nécessaires à son élimination, à une époque où l'on craignait des attentats dus à l'OAS. Le LERA est une sorte de pendant aux laboratoires de radioécologie, mais tourné vers des études portant sur l'irradiation et la contamination interne de gros animaux : ingestion, élimination, transferts aux différents tissus et organes, notamment pour les animaux d'élevage qui constituent un maillon des chaînes alimentaires pour les produits carnés et laitiers. De son côté, l'INRA mène des expérimentations utilisant des indicateurs radioactifs et des molécules marquées, dans certains 
domaines comme la physiologie de la reproduction, de la croissance ou de la lactation.

(e) On se gardera d'oublier la partie essentielle de tous ces travaux, c'est-à-dire la radioécologie elle-même, qui, balbutiante avant 1960, a vraiment pris son essor entre 1960 et 1965. En 1960, le Pr Y. Fontaine (Museum) dresse un premier bilan de la contamination radioactive des milieux et des organismes aquatiques (Fontaine, 1960). Des études de transfert en aquarium sont entreprises et des relevés écologiques sont réalisés en mer, sur le site de La Hague. Débutent aussi les études de dispersion des effluents à partir de simulations, à l'aide de lâchers de cartes et de bouées et de rejets de colorants. Des études socio-économiques permettent de connaître les pratiques relatives à la pêche, au ramassage des coquillages et aux habitudes alimentaires de divers groupes de population concernés (Ancellin, 1964). En 1964, le colloque de radioécologie marine de Cherbourg rassemble de nombreuses présentations françaises et étrangères.

À Cadarache, J. Delmas et L. Foulquier lancent la radioécologie continentale. L. Foulquier prépare une thèse de spécialité sur "étude expérimentale de la contamination de Margaritana margaritifera par le ${ }^{137} \mathrm{Cs} »$; ce bivalve d'eau douce a été identifié comme étant un excellent marqueur, lors de l'étude du site de la centrale de Brennilis; il a été utilisé par la suite pour l'étude du site de Cadarache. Ces recherches donneront lieu à une publication (Foulquier et al., 1966). Comme pour le site de La Hague, le site de Cadarache exige des études préliminaires permettant de faire le point écologique et radiologique. L'équipement devient de plus en plus performant pour la réalisations d'expérimentations diverses sur les sols, les cultures, les eaux, la flore et la faune aquatiques.

\section{Conclusion}

L'histoire de la naissance et des premiers développements des études radioécologiques en France a été reconstituée avec quelques difficultés qui tiennent à l'absence de la plupart des acteurs qui en furent à l'origine, et au peu d'archives disponibles.

Lorsque l'on compare à la situation actuelle ce que fut la radioécologie à ses origines, on ne peut qu'être frappé par l'ampleur du chemin parcouru. On observe aussi que les grands axes, sur lesquels ont été lancées les premières études, sont en assez bonne concordance avec ceux qui, tout au long des décades suivantes, ont conduit à cette situation. Les conséquences de la mise en œuvre de programmes nucléaires militaires et la perspective de celle de programmes nucléaires civils, l'émergence des problèmes de pollution associés à la révolution industrielle de 
l'après-guerre, enfin les craintes suscitées dans le milieu agricole, sont autant de moteurs qui ont poussé le développement de la prise en compte de l'environnement vis-à-vis de la contamination radioactive, donc de la radioécologie.

Ce fut une belle aventure humaine avant de devenir, plus prosaïquement, « la science en marche », avec - la suite le montrera - une grande réussite vis-à-vis de la protection de la population, même s'il n'est pas toujours facile d'en convaincre le public.

Remerciements. L'auteur remercie vivement G. Michon, qui a contribué largement à reconstituer une grande partie des évènements décrits, ainsi que L. Foulquier et A. Grauby, pour leur aide précieuse.

\section{Notes importantes}

Aux références, présentées selon les instructions aux auteurs, s'ajoute une liste de documents non-publiés, cités dans le texte. Ces documents peuvent être consultés au service des archives du CEA, centre d'études de Fontenay-aux-Roses. Voici ces documents : [a] note CEA B 41, 6 novembre 1951; [b] note CEA B 300, 6 avril 1956 ; $[c]$ note CEA 56-359, 19 juin 1956; [d] note CEA du 23 juillet 1957 ; [e] note CEA C 480, 31 juillet 1961 ; [f] note CEA C 481, 31 juillet 1961 ; $[\mathrm{g}$ ] note CEA C 584, 20 février 1962 ; [ $h]$ note CEA C 583, 20 février 1962 ; [i] note CEA du 21 mars 1963 ; [j] note CEA C 524, 29 janvier 1962 ; [ $k$ ] note CEA C 946, 26 novembre 1964 ; [l] circulaire CEA/DPS/65.9.D, 9 février $1965 ;[\mathrm{m}]$ note CEA/DPS/65.389 Dir, 11 février 1965; [n] circulaire CEA/DPS/65.11.D, 9 février 1965 ; [o] circulaire CEA/DPS/65.42.D, $1^{\text {er }}$ septembre $1965 ;[p]$ circulaire CEA/DPS/65.43.D, $I^{\text {er }}$ septembre $1965 ;[q]$ note de service CEA/C 1137, 26 janvier 1966; [r] note de service CEA/C 1138, 26 janvier 1966.

\section{RÉFÉRENCES}

Académie d'agriculture de France (1958) Les problèmes généraux de la pollution chimique sur les champs cultivés et dans les eaux, séance du 25 juin.

Académie d'agriculture de France (1963) Étude expérimentale de la contamination radioactive des cultures notamment par l'eau d'irrigation, séance du 8 mai.

Ancellin J. (1964) Facteurs socio-économiques et humains intervenant dans le rejet d'effluents radioactifs en milieu marin, Colloque de radioécologie marine, Cherbourg, 22-25 avril.

Barbier G., Michon G. (sous la direction de) (1959) Sur la prévision de l'accumulation dans le sol et dans les récoltes, d'éléments radioactifs de longue période apportés indéfiniment à un rythme

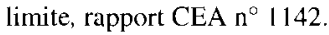

Barbier G, Michon G. (1960) Rejets d'effluents faiblement radioactifs et accumulation dans les sols cultivés, AIEA, disposal of radioactive wastes, Monaco, 16-21 novembre.

Bourrier J., Bovard P, Grauby A. (1960a) Mesure pratique de la vocation des sols à la rétention des radioéléments, rapport CEA $\mathrm{n}^{\circ} 1557$. 


\section{R. COULON}

Bourrier J., Bovard P., Grauby A. (1960b) Méthode d'étude de la contamination des sols en place par les radioéléments, bulletin technique du génie rural, $\mathrm{n}^{\circ} 48$, septembre.

Bovard P., Grauby A. (1960) Étude et contrôle de la contamination des sols cultivés, note CEA n 320.

Bovard P, Grauby A. (1962) Mesure en continu de la radioactivité de l'eau, Chim. Analyt. 44(10), 439-443.

Bovard P., Grauby A., Boyer J. (1961) Rétention et accumulation du ruthénium-106 dans les échantillons de divers types de sols, rapport interne CEA.

Coulon R. (1966) Conception et réalisation d'un contrôle de la pollution radioactive de la châne alimentaire, IRPA, $I^{e r}$ congrès de radioprotection, Rome, 5-10 septembre, Vol. 2, 1035-1038.

Coulon R. (1969) Mécanismes de la contamination radioactive des céréales. Conséquences sur la pollution de la farine, Symposium international de radioécologie, Cadarache, 8-12 septembre, pp. 833-862.

Coulon R. (1972) Deposition of ${ }^{90} \mathrm{Sr}$ and contamination of milk. Proposition of a model of transfert, 2nd international conference on strontium metabolism, Glasgow, 16-18 août.

Coulon R. (1992) Les essais nucléaires atmosphériques dans le monde. Conséquences radiologiques pour l'environnement et la population, R.G.E. 2, 136-139.

Coulon R., Madelmont C. (1969) Étude comparée chez l'homme du rapport ${ }^{90} \mathrm{Sr} / \mathrm{Ca}$ dans l'alimentation et le tissu osseux, rapport CEA R-3848.

Coulon R., Jeanmaire L., Michon G. (1963) Méthodes d'étude de la contamination radioactive des végétaux, rapport CEA n ${ }^{\circ} 2339$.

Daburon J. et al. (1987) Transfert à la viande et au lait de vache des radiocésiums présents dans la ration alimentaire, Colloque de la fondation française pour la nutrition, Paris, 11 juin.

Ferron F. (1964) Absorption du césium par l'orge. Influence de sa rétention dans le sol. Action compétitive du potassium, Ann. Physiol. Végét. 6(2), 91-117.

Fontaine Y. (1960) La contamination radioactive des milieux et des organismes aquatiques, rapport CEA $n^{\circ} 1588$.

Foulquier L. (2000) Radioécologie, techniques de l'ingénieur, BN 3 908, 3-4.

Foulquier L., Bovard P., Grauby A. (1966) Contamination expérimentale de Margaritana margaritifera (L), bivalve d'eau douce, par ${ }^{137} \mathrm{Cs}$, rapport CEA R 3054.

Grauby A. (2002) communication personnelle.

INRA/SHARP (1961) Absorption de radioéléments du sol par divers légumes cultivés dans des conditions de la pratique, rapport CEA $\mathrm{n}^{\circ} 1860$.

INRA/DPS (1962) Compte rendu d'expériences de plusieurs années sur l'absorption du strontium et du césium radioactifs par les plantes cultivées, rapport CEA n ${ }^{\circ} 2159$.

Michon G., Jeanmaire L. (1963) Essai d'étude de la contamination du cheptel en période de retombées radioactives, rapport CEA $n^{\circ} 2323$.

UNSCEAR (1958) Sources et effets, rapport à l'assemblée générale et annexes, ONU, New-York. 